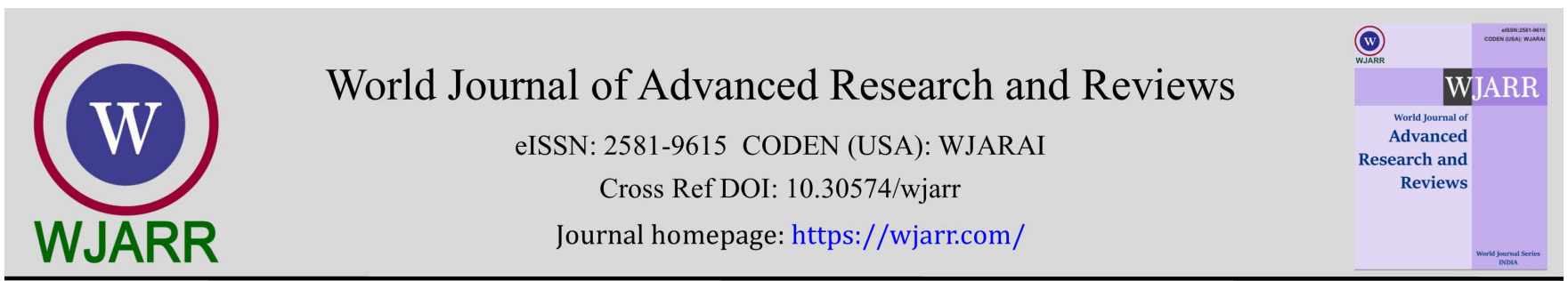

(REVIEW ARTICLE)

Check for updates

\title{
Nutrition role in the well-being of people during Covid 19 pandemic-A review article
}

Abhishek Lachyan 1, ${ }^{*}$, Randhir S Gendeh a/l Jekinder Singh 1, Abubakar Mannir 2, Pooja Nilgar 3, Rafdzah Ahmad Zaki ${ }^{1}$, Bratati Banerjee ${ }^{4}$, Karan Gade 5, Rajashri Virge ${ }^{6}$, Nitin Basole ${ }^{7}$ and Neelakanthayyaswamy I Hiremath 8

${ }^{1}$ Centre for Epidemiology and Evidence-Based Practice, Department of Social and Preventive Medicine, Faculty of Medicine, University of Malaya 50603, Kuala Lumpur, Malaysia.

${ }^{2}$ Department of Public Health, School of Health Sciences, Noida International University, Delhi NCR, India.

${ }^{3}$ Department of Public Health, JNMC KAHER Belgaum, India.

${ }^{4}$ Department of Community Medicine Maulana Azad Medical College- New Delhi. India.

${ }^{5}$ Indian Institute of Public Health, Gandhinagar, Gujarat, India.

${ }^{6}$ National Institutes of Traditional Medicine, Belagavi, Karnataka, India.

${ }^{7}$ Integrated Disease Surveillance Programme Unit, National Health Mission, Nanded-431601, Maharashtra, India.

${ }^{8}$ Women's and Children's Health Research Unit, J N Medical College, KAHER, Belgaum 590010 Karnataka, India.

World Journal of Advanced Research and Reviews, 2021, 11(02), 237-240

Publication history: Received on 11 July 2021; revised on 14 August 2021; accepted on 16 August 2021

Article DOI: https://doi.org/10.30574/wjarr.2021.11.2.0381

\begin{abstract}
The worldwide health catastrophe caused by the Coronavirus Disease 2019 (COVID-19) pandemic has startled world health experts. Obesity and poor physical activity levels were shown to be elevated risk factors for COVID-19 affection and physiopathology, indicating that the COVID-19 lockdown encouraged harmful dietary changes and increases in body weight in the community. Furthermore, malnutrition and deficiencies in vitamin C, D, B12, selenium, iron, omega3 , and medium and long-chain fatty acids were found in hospitalized COVID-19 patients, emphasizing the potential health benefits of vitamin $\mathrm{C}$ and $\mathrm{D}$ treatments. More research is needed to determine the full function and consequences of diet in the prevention and treatment of COVID-19 infected individuals.
\end{abstract}

Keywords: Nutrition; Diet; COVID -19; Pandemic; Well-being

\section{Introduction}

From December 2019, the coronavirus illness (COVID-19) has swiftly spread throughout the world, creating a pandemic, according to the World Health Organization (WHO). Millions of infections have been documented, resulting in numerous fatalities across the world. COVID-19 is caused by the coronavirus disease (COVID-19), which is caused by the severe acute respiratory syndrome coronavirus 2 (SARS-CoV-2). It is a quickly spreading disease [1]. People of all ages are infected with COVID-19. Evidence shows that two categories of persons are more likely to have severe COVID19 disease. People over the age of 40, as well as those with underlying medical issues, fall into this category [2,3]. Aside from boredom, constant media coverage of the COVID-19 can be stressful. Stress causes people to overeat, particularly 'comfort foods,' which are mostly high in sugar, a condition known as "food craving." These foods, which are mostly high in simple carbs, can help to relieve stress by promoting serotonin production, which has a good influence on mood. Beyond a chronic state of inflammation, which has been shown to increase the risk for more severe COVID-19 and other health conditions, this food craving effect of carbohydrates is proportional to the glycaemic index of foods that are associated with an increased risk of developing obesity and cardiovascular diseases [4].

\footnotetext{
${ }^{*}$ Corresponding author: Abhishek Lachyan

Centre for Epidemiology and Evidence-Based Practice, Department of Social and Preventive Medicine, Faculty of Medicine, University of Malaya 50603, Kuala Lumpur, Malaysia.

Copyright $(2021$ Author(s) retain the copyright of this article. This article is published under the terms of the Creative Commons Attribution Liscense 4.0.
} 
COVID-19 can't be caught via food, including fruits and vegetables, according to current research. Fresh fruits and vegetables are an important element of a balanced diet and should be consumed regularly. Fruit and vegetables should be washed the same way they would be washed in any other situation. Hands should be washed with soap and water before handling them. Then, especially if you're eating raw fruits and veggies, carefully wash them with clean water. Coronaviruses cannot proliferate and survive on the surface of food containers because they require a living animal or human host to reproduce and thrive. Food packing materials do not need to be disinfected, but hands should be thoroughly cleaned after handling food packages and before eating. There is presently no indication that COVID-19 can be contracted through food. COVID-19 is a virus that can be destroyed at temperatures comparable to those used to kill other viruses and germs found in food. Meat, poultry, and eggs should always be properly cooked to a temperature of at least $70^{\circ} \mathrm{C}$. Raw animal products should be handled with caution before cooking to avoid cross-contamination with cooked meals. Yes, going grocery shopping and to markets is typically safe if you take the precautions listed below: Before entering the store, wash your hands with sanitizer. Cover a cough or sneeze with a tissue or your bent elbow. Maintain a 1-meter separation from people, and if you can't keep this distance, use a mask (many stores now require a mask). When you go home, wash your hands thoroughly, as well as after handling and storing your new purchases. COVID-19 has not yet been confirmed to be transmitted through food or food packaging. Yes, having groceries delivered is safe if the delivery service maintains appropriate personal and food hygiene procedures. Hands should be properly cleaned after accepting food/grocery deliveries. The virus may be efficiently removed from domestic surfaces with regular washing and disinfection solutions. Surface veridical disinfectants, such as 0.05 percent sodium hypochlorite ( $\mathrm{NaClO}$ ) and products based on ethanol (at least 70\%), should be used to clean and disinfect houses with suspected or proven COVID19. Many nutrients are required to sustain the immune system. For a healthy and balanced diet, a range of foods such as whole grains, legumes, vegetables, fruits, nuts, and animal source foods should be consumed. There is no single meal that can protect you from COVID-19 infection. There is presently no recommendation on the use of micronutrient supplementation to prevent COVID-19 in healthy people or to treat COVID-19. Micronutrients are essential for a healthy immune system and play an important part in overall health and nutrition. Micronutrients should be obtained as much as possible through a nutritionally balanced and diversified diet, which includes fruits, vegetables, and animal source meals. Vitamin D is produced in the skin by exposure to sunshine, or it can be acquired through the diet from natural sources (fatty fishes like salmon, tuna, and mackerel, fish liver oils, cow liver, cheese, and egg yolks), vitamin D-fortified foods, or vitamin D-containing pills.

In situations where individuals' vitamin D status is already marginal or where foods rich in vitamin D (including vitamin D-fortified foods) are not consumed, and exposure to sunlight is limited, a vitamin D supplement in doses of the recommended nutrient intakes (200-600 IU, depending on age) or according to national guidelines may be considered. There is currently no evidence to support the use of herbal teas or herbal supplements to prevent or cure COVID-19. Probiotics are live microorganisms that are generally added to foods or used as a supplement to the diet to confer a health benefit. However, there is currently no evidence to support the use of probiotics to help prevent or cure COVID19. There is no evidence that eating ginger has protected people from COVID-19. However, ginger is a food that may have some antimicrobial and anti-inflammatory properties. There is no evidence that eating garlic has protected people from COVID-19. However, garlic is a food that may have some antimicrobial properties. There is no evidence that adding hot peppers to your food can prevent or cure COVID-19.

It is critical to satisfy daily dietary needs in order for one's body to function properly and for one's health to be at its best. The majority of nutritional qualities, such as protein, energy, carbs, fats, and minerals, may be acquired through the foods we eat. Some people, on the other hand, use dietary supplements on a regular basis to guarantee enough nourishment. Each vitamin serves a critical role in maintaining bodily health, metabolism, and appropriate function [5].

Food is divided into six categories: carbs, fats and oils, protein, vitamins, minerals, and water. All of these food groups are necessary for an individual's growth, and a balanced diet is one that includes all of them in the correct proportions in a meal. Poor nutrition occurs when one or two types of food are consumed in excess or in insufficient quantities, resulting in sickness. Malnutrition is a nutritional condition in which a lack of, excess, or imbalance of energy, protein, and other nutrients has quantifiable negative consequences on tissue or body form (size, shape, and composition), function, and clinical outcome. It encompasses both overnutrition (too many nutrients) and undernutrition (not enough nutrients) (insufficient nutrition) [6].

\section{Roles of good nutrition on one's well - being}

Nutrition has a critical function throughout the life cycle. Food, water, and oxygen are required for survival. Food provides the body with both the energy it requires to stay alive and the nutrients it requires to build and repair all of its cells. And nutrients travel through a complicated process throughout the body, cells, and tissues to sustain an individual's general health. Nutritional demands must be met at all stages of human life, from conception to growth and 
development, and in order to ensure long-term survival [7]. Diet and lifestyle are modifiable risk factors that have a major influence on an individual's likelihood of developing an NCD. It has also become clear that one's nutritional state is a key component in preparing the human immune system to respond to acute infections like COVID-19. Healthy eating habits can help to avoid NCDs and boost the immune system, which can help to lessen the severity of an infection [8]

People of all ages can work successfully and feel their best when they eat a nutritious diet. A well-balanced diet will offer you with a variety of vitamins and minerals that work as co-enzymes, or enzymes that speed up chemical reactions in the body. A balanced diet will give your body with the calcium it requires to keep proteins, which are components of your immune system, hormones, neurological system, and organs, in good working order. Phytochemicals, which are present in the colorful portions of fruits and vegetables and have a strong influence on your health, are provided by a well-balanced diet. Antioxidants can be found in the phytochemicals of fruits and vegetables, as well as certain vitamins and amino acids, in a balanced diet [6]

\section{COVID 19 on nutrition and wellbeing}

Nutrition is not a treatment for COVID-19, but it does enhance immune system function and is a controllable factor to the development of chronic illness, which is strongly linked to COVID-19 fatalities. COVID-19 may also benefit from nutrition since it can aid those who are at higher risk for the illness, such as the elderly and those with pre-existing diseases (NCDs) [8] People who consume a well-balanced diet are healthier and have a decreased risk of obesity and its consequences. They will also have better immune systems and be less likely to develop infectious illnesses such as COVID-19 [7].

COVID-19 management methods like as healthy diet and nutrition have been proven to have an influence on the immune system and health consequences. A well-balanced diet can help the immune system fight viral infections. Healthy meals have been identified as a possible treatment for improving immunity, acute respiratory symptoms, and health outcomes, all of which may aid in protecting individuals during the COVID-19 pandemic.[8] Some dietary categories and minerals have been found to be beneficial in preventing viral infection. Adequate nutritional intake can assist to reduce inflammation and oxidative stress, which can help to boost people's immune systems during the COVID-19 pandemic [9].

The most effective method for reducing the ongoing risk of viral infection is to optimize nutritional intake through wellbalanced meals and to adopt appropriate hygienic standards in food selection, preparation, and storage [10,11].

WHO nutrition advises during COVID 19 outbreak [12,13].

- $\quad$ Eat fresh and unprocessed foods everyday

- Drink enough water everyday

- Eat moderate amount of fat and oil

- $\quad$ Eat less salt and sugars

\section{Conclusion}

To summarize, while there is still much to learn about COVID-19, its impact on nutrition and food intake has already spread beyond the individual and community to national and global levels. The interconnectedness of these many levels was highlighted by this epidemic, whereby an individual's health became a direct consequence of his own understanding and choices, community unity, government readiness, and, eventually, global participation in response to this threat. Obesity and physical activity levels were shown to be risk factors for COVID-19 in the current investigation, indicating that the COVID-19 lockdown encouraged harmful dietary choices and increases in body weight in the community. Furthermore, malnutrition and deficiencies in vitamin C, D, B12, selenium, iron, $\omega-3$, and medium and long-chain fatty acids were found in hospitalized COVID-19 patients, emphasizing the potential health benefits of vitamin C and D treatments. More research is needed to determine the full function and consequences of diet in both the prevention and treatment of COVID-19 patients.

\section{Compliance with ethical standards}

\section{Acknowledgments}

There was no particular grant for this research from any funding agency in the public, private, or non-profit sectors. 


\section{Disclosure of conflict of interest}

The authors declare that they have no competing interests.

\section{Statement of informed consent}

There are no potential conflicts of interest.

\section{References}

[1] Tsoupras A, Lordan R and Zabetakis I. Thrombosis and COVID-19: The Potential Role of Nutrition. 2020.

[2] World Health Organization. (0) Coronavirus disease 2019 .(19-COVIDWorld Health .situation report, 51 : .Organization. 2020.

[3] Aritra Ghosh, Srijita Nundy, Tapas K. Mallick. How India is dealing with COVID-19 pandemic.

[4] Nasih O, Simon NH, Lachyan AS. A comparative study of nutritional status of urban and rural schoolchildren in selected developing countries: systematic review. Language. 2015; 2015(2021).

[5] Di Renzo L, Gualtieri P, Pivari F, Soldati L, Attinà A, Cinelli G, Leggeri C, Caparello G, Barrea L, Scerbo F, Esposito E. Eating habits and lifestyle changes during COVID-19 lockdown: an Italian survey. Journal of translational medicine. 2020 Dec;18:1-5.

[6] Mannir A, Lachyan AS, Simon NH. Systematic Review on Assess the Knowledge, Attitude, and Practice of Nutrition among Undergraduate Students.

[7] María Belén Ruiz-Roso, Patricia de Carvalho Padilha, Diana C. Mantilla Escalante, Natalia Ulloa, Paola Brun, Diofanor Acevedo-Correa, Wilza Arantes Ferreira Peres, Miquel Martorell, Mariana Tschoepke Aires, Letícia de Oliveira Cardoso, Fernanda Carrasco-Marín, Katherine Paternina-Sierra, Jhon E. Rodriguez-Meza, Piedad M. Montero, Giulia Bernabè, Anthony Pauletto, Xhoajda Taci, Francesco Visioli, and Alberto Dávalos. Covid-19 Confinement and Changes of Adolescent's Dietary Trends in Italy, Spain, Chile, Colombia and Brazil. Received: 26 May 2020.

[8] Emmanuel O.Umenweke, Onyinye N. Umenweke \& Chinwe I. Onyemaechi, Health and Nutrition across the Lifespan. 2017.

[9] e: Matsungo TM, Chopera P. Effect of the COVID-19-induced lockdown on nutrition, health and lifestyle patterns among adults in Zimbabwe. BMJ Nutrition, Prevention \& Health. 2020.

[10] Mannir, A., Lachyan, A. S., \& Simon, N. H. (2021). Food literacy programmes in general population: a systematic literature review.

[11] Naja, F., Hamadeh, R. Nutrition amid the COVID-19 pandemic: a multi-level framework for action. Eur J Clin Nutr. 2020; 74: 1117-1121.

[12] Pham KM, Pham LV, Phan DT, Tran TV, Nguyen HC, Nguyen MH, Nguyen HC, Ha TH, Dao HK, Nguyen PB, Trinh MV, Do TV, Nguyen HQ, Nguyen TTP, Nguyen NPT, Tran CQ, Tran KV, Duong TT, Nguyen LV, Do TT, Vo TT, Do BN, Duong TH, Pham TTM, Le TT, Do NT, Nguyen HTT, Mai TTT, Ha DT, Ngo HTM, Nguyen KT, Yang S-H, Chao JC-J and Duong TV (2020) Healthy Dietary Intake Behavior Potentially Modifies the Negative Effect of COVID-19 Lockdown on Depression: A Hospital and Health Center SurveyKaren Danna and Ricky W. Griffin, Health and Well-Being in the Workplace: A Review and Synthesis of the Literature, First.

[13] Christianne de Faria Coelho-Ravagnani, Flavia Campos Corgosinho, Fabiane La Flor Ziegler Sanches, Carla Marques Maia Prado, Alessandro Laviano, and Jo ao Felipe Mota, Dietary recommendations during the COVID19 pandemic. 